\title{
Recent advances, approaches and challenges in targeting pathways for potential COVID-19 vaccines development
}

\author{
Daniela Calina ${ }^{1} \cdot$ Chandan Sarkar $^{2} \cdot$ Andreea Letitia Arsene $^{3} \cdot$ Bahare Salehi $^{4,5}$ - Anca Oana Docea ${ }^{6}$ (D) \\ Milon Mondal $^{2} \cdot$ Muhammad Torequl Islam $^{2} \cdot$ Alireza Zali $^{7} \cdot$ Javad Sharifi-Rad $^{8}$
}

Received: 2 September 2020 / Accepted: 11 September 2020 / Published online: 1 October 2020

(C) Springer Science+Business Media, LLC, part of Springer Nature 2020

\begin{abstract}
During the COVID-19 pandemic in a modern era, there is a global consensus on the need for the rapid development of a vaccine against SARS-CoV-2 for effective and sustainable control. Developing these vaccines is fundamental to public health. This urgent need is supported by the scientific explosion in structural and genomic biology that facilitates the urgent development of an ideal COVID-19 vaccine, using new pathways to facilitate its large-scale development, testing, and manufacture. Here, we summarize the types of COVID-19 candidate vaccines, their current stage in early testing in human clinical trials, and the challenges for their implementation.
\end{abstract}

Keywords SARS-CoV-2 $\cdot$ COVID-19 pandemic $\cdot$ Immunology $\cdot$ Candidatevaccines $\cdot$ Safety $\cdot$ Efficacy $\cdot$ Quality $\cdot$ Publichealth

\section{Introduction}

In late December 2019, information emerged about a mysterious pneumonia in Wuhan, a city in Hubei, a Chinese province. Six months later, in the context of more than ten million cases, the COVID-19 pandemic has become the worst public health crisis of the last century [1].

A number of coronaviruses (HCoV-229E, HCoV-NL63, HCoV-HKU1, HCoV-OC43) cause endemic mild respiratory

Daniela Calina

calinadaniela@gmail.com

$\triangle$ Anca Oana Docea

daoana00@gmail.com

Javad Sharifi-Rad

javad.sharifirad@gmail.com

Chandan Sarkar

chandan.sarkar@gmail.com

Andreea Letitia Arsene

andreea.arsene@umfcd.ro

Milon Mondal

milonmondal18@gmail.com

Muhammad Torequl Islam

muhammad.torequl.islam@tdtu.edu.vn

Alireza Zali

dr_alirezazali@yahoo.com conditions, in the group of common "colds" or "common respiratory viruses." Two other coronaviruses caused severe respiratory epidemics.

SARS-CoV identified in 2002 in southern China, in the rapidly spreading global region of Gunagdong and which by the time of its disappearance in 2004, caused over $8000 \mathrm{hu}-$ man cases and 774 deaths (9.5\% mortality) [2]. MERS-CoV (Middle East Coronavirus Respiratory Syndrome) was identified in 2012 in Saudi Arabia, later spread to 27 countries and it

1 Department of Clinical Pharmacy, University of Medicine and Pharmacy of Craiova, 200349 Craiova, Romania

2 Department of Pharmacy, Bangabandhu Sheikh Mujibur Rahman Science and Technology University, Gopalganj 8100, Bangladesh

3 Department of Department of Microbiology, Carol Davila University of Medicine and Pharmacy, Bucharest, Romania

4 Noncommunicable Diseases Research Center, Bam University of Medical Sciences, Bam, Iran

5 Student Research Committee, School of Medicine, Bam University of Medical Sciences, Bam, Iran

6 Department of Toxicology, University of Medicine and Pharmacy of Craiova, 200349 Craiova, Romania

7 Functional Neurosurgery Research Center, Shohada Tajrish Neurosurgical Comprehensive Center of Excellence, Shahid Beheshti University of Medical Sciences, Tehran 02125719, Iran

8 Phytochemistry Research Center, Shahid Beheshti University of Medical Sciences, Tehran, Iran 
is still circulating in the Middle East. MERS-CoV has so far resulted in 2521 diseases with gastrointestinal symptoms, acute renal failure and 919 deaths (35\% mortality), associating gastrointestinal symptoms and acute renal failure. SARS$\mathrm{CoV}-2$ is one of the many species of coronavirides that predominantly infect animals but sometimes humans. Four other species of human coronaviruses are known to cause seasonal rhinopharyngitis but also two zoonotic species that cause severe respiratory infections with high mortality (SARS and MERS). The virus is an infectious agent that can only multiply with the help of a cell. It consists of genetic material (DNA or RNA) that encodes the structure of viral proteins; capsid, protein coat that protects the genetic material; and lipid tire with a protective role (maybe missing) [2].

Coronavirus is a positive-stranded RNA-type singlestranded virus (+ ssRNA) viral species. Protein modeling experiments on the $\mathrm{S}$ protein of the virus suggest that it has sufficient affinity for the angiotensin-converting enzyme (ACE 2) receptors of human cells to use as a mechanism of cell penetration [3]. SARS-CoV-2 (like SARS-CoV-1) infects cells through the interaction of three components: (i) the Spike protein of the virus that clings to receptors, angiotensinconverting enzyme 2(ACE2), which find on the cell membrane; (ii) serine cellular proteases that facilitate this binding; and (iii) the ACE2 receptor that is expressed on many types of endothelial cells but predominantly in the lungs and intestines. All these three elements represent potential therapeutic targets for future drugs. COVID-19 cannot be prevented by the arbitrary administration of drugs, natural products, or vitamins. The only method of prevention would be a vaccine [4].

This review aims to summarize current knowledge on strategies for the development of safe, effective, and broadspectrum vaccines to prevent SARS-CoV-2 infection (Fig. 1). A comparative analysis between the research and development of common, classic vaccines with the candidate vaccines against COVID-19 was also performed. A new feature was highlighted in the development of potential vaccines against SARS-CoV-2: new repurposing idea to shortcut vaccine development time.

\section{Immunological endpoints and clinic-epidemiological context}

Despite research by scientists around the world, it is not known exactly what a protective immune response to the COVID-19 means. But an effective vaccine may be the only way out of the pandemic. Vaccination is a method of preventing many communicable diseases, which is based on the fact that the introduction into the body of a pathogen-like agent causes an immune response, similar to the natural infection, but without producing the disease. The immunity produced by vaccines involves the recognition of the vaccine-containing agent as foreign to the body, its destruction, and the formation of immune memory so that subsequent contact with the pathogen for which the body has developed immunity results in faster and more effective defense responses. The purpose of immunization by vaccination is to prevent specific infections and to limit their consequences [4]. The ideal vaccine is one that simultaneously meets the following conditions: is effective in preventing infectious disease or reducing the severity of the disease; provides durable and long-lasting protection against the disease; develops immunity with a minimum number of administrations; provides as much antigen as possible to provide extensive protection against infections; does not cause side effects or they are minimal; is stable under storage conditions, preferably without special storage conditions, throughout the shelf life; and can be produced on a large scale; it is financially accessible to the populations to which it is addressed [4].

In this COVID-19 pandemic, the objectives of vaccination against SARS-CoV-2 are:

i) Prevention of a clinically symptomatic or severe infection that would require hospitalization

ii) Prevention of a proven seroconversion infection to prevent the transmission of the disease

iii) Generating a strong neutralizing immune response, which can bind to the viral protein $\mathrm{S}$, which can prevent it from attaching to human cells

In this context, the relative contribution of different immunological responses, such as neutralizing antibodies and CD8 $+\mathrm{T}$ cells, is very important.

Anti-SARS-CoV-2 antibodies represent the immune response of the host organism to the presence of the virus. These antibodies are immunoglobulins belonging to classes A, M, G, and less often D. Previous models of serological antibody responses to viral infections have generally established the following sequence of these antibodies over time from the time of virus infection: the earliest are $\operatorname{IgA}$ and follow IgM closely, IgG-type antibodies being the last to appear and remain in high titers for a more extended period than the previous ones (IgA and IgM) [5].

For some viruses, there is a period when the antigen (the virus itself) coexists (can be identified) with antibodies, especially early IgA and IgM antibodies. Other viruses have a period of "serological window," i.e., a time interval between the disappearance of the antigen (in the blood) and the appearance of antibodies, so a time interval in which the infection (past) cannot be highlighted [6].

IgG-type antibodies are specific to the novel coronavirus and can be measured using chemiluminescence immunological methods, an automated laboratory method with superior performance to enzyme-linked immunosorbent assays. The test basically detects the body's immune response to SARSCoV-2 infection [7]. 
Fig. 1 Schematic mechanism of the neutralizing antibodies. Spike (S) protein binds to the host receptor (ACE2 receptor) and enter into the target cell through membrane fusion. The antibody targeting S protein can effectively block binding, membrane fusion, and entry of SARS-CoV-2 to the ACE2 receptor

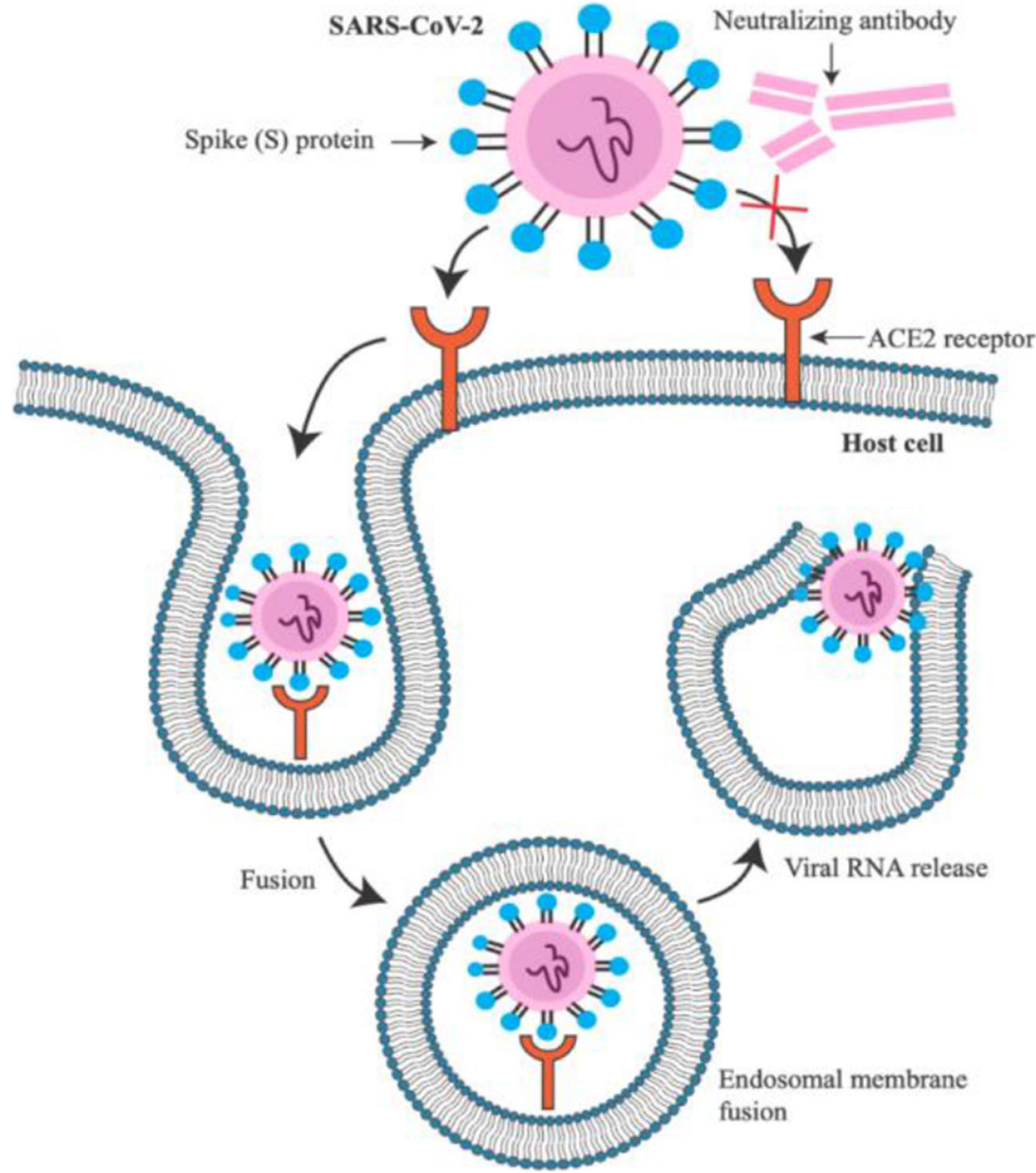

IgG-type neutralizing antibodies are formed in the body's response to viral antigens on the envelope (envelope) of the SARS-CoV-2 virus - more precisely the S1 and S2 areas (domains) of the $\mathrm{S}$ protein in that envelope. The SARSCoV-2 is an RNA-type virus that has in its structure several proteins, with the help of which the virus manages to attach to the cells of the human body, to penetrate them and to replicate and to multiply. The proteins that form the outer shell of the new coronavirus are protein $\mathrm{S}$ ( $\mathrm{S}$ comes from Spike, the characteristic, spiky appearance of the virus), protein $\mathrm{M}$, membrane protein, and protein $\mathrm{E}$ (Envelope). $\mathrm{N}$ protein is the nucleocapsid protein that envelops the viral nucleic acid [8].

These proteins are the portions of the structure of the virus that are recognized by the human body as "attackers" causing the formation of antibodies specific to the attackers (as a key suitable for a lock). Usually, the standard time for antibody development is 5-21 days after the onset of symptoms, with individual variations, IgM-type antibodies appearing earlier (usually $5-12$ days), and IgG-type antibodies later (usually 7-14 days)[9].

Neutralizing antibodies are produced by the body specifically to bind to two areas of the S protein (protein outside the virus) that are responsible for attachment to human cells (S1 area) and the fusion of the virus to the membrane. $\mathrm{S}$ protein is the main protein involved in triggering the protective immune response, so the test has a high sensitivity, being able to detect with maximum accuracy the presence of this important viral protein. Antibodies that bind specifically to the S1 and S2 areas of the viral $\mathrm{S}$ glycoprotein prevent the virus from infecting the human cell and are called neutralizing antibodies (it blocks the entry into human cells, effectively neutralizing the virus) [10] (Fig. 1).

Only certain types of antibodies developed in response to infection are neutralizing antibodies, meaning they can block the virus from infecting human cells [6].

\section{Clinical importance of dosing IgG anti-SARS-CoV-2 antibodies}

Test results are expressed quantitatively, reflecting the amount of neutralizing antibodies present, and the result may be positive, negative, or equivocal. A threshold value is also set above which it is considered that the person can become a donor of immunized plasma (because he has a higher amount of neutralizing antibodies). The presence of anti-SARS-COV2 antibodies should be correlated with clinical signs and symptoms, epidemiological investigation, and other test results [11]. 
Currently, the body's specific immune response to SARSCoV-2 infection is the subject of numerous studies. To date, there is no complete validated information on the dynamics of antibodies to the time of infection and their persistence after virus elimination and neutralizing capacity, so their ability to protect against viral infection with SARS-CoV-2 [12]. Most published studies have as a reference term for antibody dynamics, the onset of symptoms, not the timing of infection. However, serological tests have a number of limitations. Negative serological results cannot rule out SARS-COV2 infection, especially when the infection is recent. On the other hand, there is a risk of positive results due to cross-reactivity with antibodies formed as a result of infections with other coronaviruses [13].

In rare cases, certain antibodies generated by immunization may not only prevent infection but even favor an aggravated form of the disease (antibody-dependent disease enhancement, ADE). When these antibodies come in contact with the virus again, they will help it to enter the cells and cause infection [14]. ADE has been described in many viral infections (flu, dengue, Zika, etc.) but also in coronaviruses. Several animal studies have shown that some types of antiSARS and anti-MERS vaccines, although effective in generating antibodies, can lead to more severe forms of the disease when the virus is subsequently inoculated [15].

Thus, it could be suggested that the severity of COVID-19 lung damage could be explained by ADE, perhaps due to prior exposure of patients to common coronaviruses (such as 229E), which are similar in structure and therefore interact with the same antibodies [16]. The mechanism of ADE has not been confirmed for coronavirus in humans, but we need time to remove any doubt that the vaccine will not make us more vulnerable to COVID-19[17].

In animal studies, immunization tests were performed against the previous coronavirus, SARS-CoV-1, using recombinant protein components and viral vectors based on nucleic acid, as well as the passive transfer of anti-spike protein antibodies. Some vaccines add an adjuvant to boost the immune response, creating a stronger and longer-lasting immunity to infections than the vaccine alone. The use of an adjuvant may be of particular importance in a pandemic situation, as it may reduce the amount of vaccine protein required per dose, allowing multiple doses of vaccine to be produced and therefore helping to protect more people [18].

The role of $\mathrm{T}$ lymphocytes in obtaining and maintaining this immune response is unclear, which is an additional reason for the development of several types of vaccines. New research shows that many people with asymptomatic or mild symptoms of COVID-19 demonstrate T cell-mediated immunity to the new coronavirus, even if they have not tested positive for antibodies. This means that public immunity is probably higher than antibody tests suggest.

So, a high degree of safety of the vaccine is a prerequisite for its approval for human use [19]. Unlike antibodies, which bind to the SARS-CoV-2 virus and prevent it from entering cells, $\mathrm{T}$ cells are multifaceted lymphocytes. Regulatory $\mathrm{T}$ cells $(\mathrm{CD} 4+)$ signal antibodies and other defensive systems to act when they detect the presence of the virus, and cytotoxic or killer $\mathrm{T}$ cells $(\mathrm{CD} 8+)$ detect and destroy virus-infected cells [20]. The existence of $\mathrm{T}$ cells in the body of patients who have had COVID-19 is good news for a possible prolonged immunity and therefore for the development of a vaccine. A recent study shows that $100 \%$ of patients have regulatory $\mathrm{T}$ cells, and cytotoxic cells are present in $70 \%$ of them, which means that the immune system detects the virus and triggers the immune response [21].

Preclinical data for some studied vaccines has demonstrated the occurrence of exacerbated respiratory disease due to the formation of immune complexes between antibodies with low neutralization and helper T cells 2 . The COVID-19 vaccine must be effective to determine the synthesis of antibodies of a certain type, at a certain concentration (titer), and to provide protection for a reasonable time [22]. But vaccines never generate immunity in everyone who is vaccinated. For example, $2-19 \%$ [23] of people vaccinated against measles do not get a protective immune response after the first dose, and a percentage of $2-10 \%$ fail after the second dose [24]. Certainly, for any possible anti-COVID vaccine, all these variables must be carefully evaluated, and the primary immunization failures are minimized by adjusting the dose or number of administrations [25], especially since recent studies suggest that up to $6 \%$ of individuals do not acquire post-COVID immunity.

The inability of the immune system of some individuals to generate a response is not limited to measles but is described in many common types of vaccines [26]. The causes of these primary immunization failures are very complex and range from genetic and immunological factors to the quality of the vaccines themselves and the mode of administration. Age is also an important criterion, with some studies of the flu suggesting that the aging of the immune system dramatically decreases the effectiveness of vaccination [27]. Certainly, for any possible anti-COVID-19 vaccine, all these variables must be carefully evaluated, and the primary immunization failures are minimized by adjusting the dose or number of administrations, especially since recent studies suggest that up to $6 \%$ of individuals do not acquire post-COVID-19 immunity[28].

Assuming that the COVID-19 vaccine will generate an effective immune response in a sufficient number of vaccinated individuals, we need to know how long the protection will last [29]. For example, after measles vaccination, $5-12 \%$ of those who have an excellent initial response lose their protective antibody titer within a few years, a phenomenon called secondary immunization failure [30]. Another example is the Ebola vaccine (non-replicating adenoviral vector) developed in 2017 in response to recurrent epidemics in West Africa. As the vaccine needed to reach the market faster than the platforms developed during the same period by GSK and Merck, 
the phase III tests were skipped [31]. The vaccine was administrated to 500 volunteers in Sierra Leone, and although it generated an initial immune response, most of those vaccinated had significant decreases in antibodies 168 days after immunization [32].

Existing data on human coronaviruses are very few. The coronavirus 229E seems to give immunity for only 2 years [33]. Other studies suggest that anti-SARS antibodies drop fairly rapidly to levels below those that provide protection, so that recovered individuals could become vulnerable again 3 years later [34]. In the case of MERS, although the antibody titer was still high after 3 years [35] in most survivors in one study [36] in those with mild disease, it seems to disappear faster, i.e., under 18 months $[37,38]$. It also takes time to check the post-vaccination persistence of anti-COVID antibodies.

\section{Diversity of platforms and candidate vaccines against SARS-CoV-2}

According to a document published on the WHO website (www.who.int) on 7 July and June 2020, there are twentyone clinically evaluated vaccines in the landscape of candidates for the possible vaccine against COVID-19 and 139 candidate vaccines in preclinical evaluation [39]. The most promising candidate vaccines at the moment are those already being tested in humans, in different phases (Fig. 2). Some technologies are very new and therefore require more careful testing; others are old but need to be adapted for COVID-19. Each of these has advantages and disadvantages that must be overcome [40]. The platforms for developing of candidate COVID-19 vaccines are diverse:

\section{a) Nucleic-acid vaccines}

These are the fastest in development and do not require fermentation or culture. Nucleic acid-based vaccines are obtained by inserting mRNA and DNA acids into some cells of vaccinated individuals, forcing them to manufacture immunogenic viral proteins [41]. During pandemics, the use of modern sequencing techniques and reverse genetics has an important contribution to shortening the development time of a vaccine [14].

DNA vaccines (Inovio, INO-4800, a DNA plasmid vaccine with electroporation) is already used to obtain flu virus vaccines. This produces stimulation of cellular immunity by inserting a foreign DNA into the cell's genome host. They stimulate the cellular immune response compared with most vaccines that address humoral immunity. This type of vaccine preparation is hope for effective prophylaxis of HCV viral hepatitis or viral pathology high antigenic variability (influenza virus or HIV). Although it is advantageous by avoiding the introduction of a live virus strain into the human body, this type of vaccine may have potential oncogenic risks by

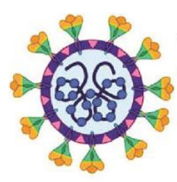

SARS-CoV-2

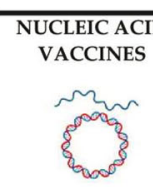

$P$

PLATFORMS

DNA

DNA plasmid vaccine with

electroporation

RNA

LNP encapsulated mRNA 3

LNP-mRNAs
CANDIDATE VACCINES

INO-4800/ USA, South Korea/ Inovio Pharmaceuticals, CEPI, Korean Institute

of Health, International Vaccine Institute

Planned Phase II-III trials

Phase I completed (two doses/ a dose every four weeks

mRNA-1273/USA/Moderna/NIAID

Phase 2 NCT04405076(IND submission)

Phase 1 NCT04283461 Immune response, Safety; 3 arms (doses 25, 100, 250 $\mathrm{mcg}) /$ Recruiting

BNT162 (a1, b1, b2, c2)/ BioNTech/ USA/Fosun Pharma/Pfizer Phase 1/2 2020-001038-36 NCT04368728

\begin{tabular}{|c|c|c|c|}
\hline $\begin{array}{l}\text { VIRAL VECTOR } \\
\text { VACCINES }\end{array}$ & $\begin{array}{l}\text { Non- Replicating } \\
\text { Adenovirus Type } 5 \text { Vector }\end{array}$ & $\begin{array}{l}\text { Ad5-nCoV/China/CanSino Biological Inc./Beijing Institute of Biotechnology } \\
\text { Phase } 2 \text { ChiCTR2000031781/Immune response, Safety; n=500; Randomized } \\
\text { double-blind placebo controlled/ Recruiting } \\
\text { Phase } 1 \text { ChiCTR2000030906/, Safety; n=108, completed } \\
\text { ChAdOx1 nCoV-19/UK/University of Oxford } \\
\text { Phase } 3 \underline{\text { ISRCTN89951424, }} \\
\text { Phase2b/3 2020-001228-32 } \\
\text { Phase } 1 / 2 \text { PACTR202006922165132 2020-001072-15 Immune response, safety, } \\
\text { n=510; Single-blinded randomized placebo controlled multicenter, completed }\end{array}$ & $\begin{array}{l}\text { Single dose } \\
\text { Medium speed } \\
\text { Licensed technology } \\
\text { Scale: high } \\
\text { requires booster shots for } \\
\text { long-term immunity } \\
\text { Safe }\end{array}$ \\
\hline $\begin{array}{c}\text { VIRUS } \\
\text { VACCINES } \\
\end{array}$ & $\begin{array}{l}\text { Attenuated } \\
\text { Codon deoptimized live } \\
\text { attenuated vaccines } \\
\text { Inactivated } \\
\text { Inactivated SARS-CoV + Alum } \\
\text { (Inactivated) }\end{array}$ & $\begin{array}{l}\text { Pre-clinical stage } \\
\text { Codagenix/Serum Institute of India } \\
\text { Indian Immunologicals Ltd/Griffith University } \\
\text { PiCoVacc (Sinovac Biotech) } \\
\text { Phase } 3 \text { NCT04456595 } \\
\text { Phase } 1 / 2 \text { NCT04383574 NCT } 04352608 \\
\text { Immune response, safety, } \mathrm{n}=144, \text { Randomized double-blind single-center } \\
\text { placebo-controlled, completed }\end{array}$ & $\begin{array}{l}\text { Single-dose, Slow speed } \\
\text { Licensed technology } \\
\text { Scale: high, Inexpensive } \\
\text { Single-dose, Fast speed } \\
\text { Not licensed technology } \\
\text { Scale: medium to high } \\
\text { Needs adjuvants for immune } \\
\text { response }\end{array}$ \\
\hline $\begin{array}{l}\text { PROTEIN-BASED } \\
\text { VACCINES }\end{array}$ & $\begin{array}{l}\text { Subunit } \\
\text { Full-length recombinant SARS } \\
\text { CoV-2 glycoprotein } \\
\text { nanoparticle vaccine adjuvanted } \\
\text { with Matrix M }\end{array}$ & $\begin{array}{l}\text { Clover Biopharmaceuticals Inc/GSK; Vaxil Bio, AJ Vaccines } \\
\text { Genrex/EpiVax/University of Georgia; Sanofi Pasteur; Novavax } \\
\text { Heat Biologics/University of Miami } \\
\text { University of Queensland/GSK/ Baylor College of Medicine; iBio/CC- } \\
\text { Pharming }\end{array}$ & $\begin{array}{l}\text { Multiple doses } \\
\text { Licensed technology } \\
\text { Medium to fast speed } \\
\text { Scale: high } \\
\text { Safe }\end{array}$ \\
\hline
\end{tabular}

Fig. 2 Vaccines platforms and current status of potential candidate COVID-19 vaccines
COMMENTS

Multiple doses

Fast speed

Not licensed technology

Scale: low to medium

The skewed response of $\mathrm{T}$ helper $\left(\mathrm{TH}_{2}\right)$ cells

Safe

Single do

Scale: high

requires booster shots fo

long-term immunity

Single-dose, Slow speed ed technology

Needs adjuvants for immune

icensed technology

Scale: high 
incorporating DNA into the chromosomes of the host cell or by inhibiting tumour suppressor genes [42].

RNA vaccines (Moderna, BioNTech/Pfizer, CureVac) are new and have no approval, but there are candidate vaccines for COVID-19. These are vaccines that try to cause the production of antibodies against the viral protein - spike - that is found on the surface of the virus. These antibodies have a neutralizing effect and block the protein that allows the infection of cells in the respiratory tract [41].

\section{b) Non-replicating viral vectors vaccines}

These common viruses (e.g., adenoviruses) have been genetically modified to express SARS-CoV proteins on the outer surface. But being common, most people have come in contact with them, and there is already an immunity to them, and they are quickly neutralized [43]. The candidates vaccines are: ChAdOx1 nCoV-19 (currently known as AZD1222)/University of Oxford/AstraZeneca/ Phase3; ISRCTN89951424/University of Oxford/ Phase2b/3; 2020-001228-32/UK-MHRA/Phase1/2; PACTR202006922165132/University of Oxford/ AstraZeneca/Phase1/2; 2020-001072-15/University of Oxford/AstraZeneca/Phase1/2). This uses a viral vector based on a weakened version of the common cold virus (adenovirus) that contains the genetic material of the surface spike (S) protein of SARS-CoV-2. After vaccination, protein $\mathrm{S}$ is produced, which causes the body's immune system to attack the new coronavirus if it infects the vaccinated person in the future with antibodies specific to this protein. The recombinant adenovirus vector (ChAdOx1) was chosen because it generates a strong single-dose immune response and does not reproduce, so it cannot cause a continuous infection in the body that received the vaccine. ChAdOx1 vaccines have been given to more than 320 people to date and are safe and well-tolerated. However, they can cause temporary side effects such as high body temperature, flu-like symptoms, headaches, or pain in the arm in which it is injected. There are used the same platforms as for MERS, influenza, TB, Chikungunya, Zika, MenB, and plague.

Ad5-CoV (phase 2 ChiCTR2000031781, phase 1 ChiCTR2000030906) Ad5-nCoV is a new, genetically engineered vaccine with replication of type 5 adenovirus as a vector of immunity against the new coronavirus protein. The vaccine tries to teach the body to recognize the $S$ protein of the coronavirus, which is delivered via a type 5 adenovirus. The potential candidate is developed by the Chinese Military Academy of Medical Sciences and Cansino Biologics and is based on the technological platform developed by Cansino which related to viral adenoviruses. It is the same platform where an Ebola vaccine was succeeded in 2017 [44]. c) Virus vaccines

Live attenuated viruses can be considered variants of SARS-CoV-2 with pathogenesis greatly diminished by genetic engineering. They are the most potent immunogenic vaccines, but there is a risk of them becoming pathogenic again as a result of mutations, as happened with the polio vaccine. They contain live microorganisms, with low virulence through repeated transitions on culture media, through passages to different animal hosts, or through genetic mutations. It provides persistent protection similar to that natural postinfection but may cause multiple and severe post-vaccination reactions. Such preparations are $\mathrm{BCG}$ and most antiviral vaccines - polio with live strains, anti-measles, and rubella [45].

Inactivated viruses containing whole bacterial/viral particles are inactivated (killed) by heat or formalization. Induced post-vaccinal immunity is lower compared with the determination of the previous preparations, but also, the side effects are lower. This category includes pertussis vaccines cellular, inactivated anti-hepatitis A, or antipoliomyelitis [46].

\section{d) Protein-based vaccines}

Subunit vaccines - with antigenic fragments - Novavax, NVX-CoV2373, phase 1/2 NCT04368988, is a vaccine based on glycoprotein nanoparticles. Matrix $\mathrm{M}$ adjuvant is used to increase the immune response against the SARS-CoV-2 spike protein by inducing elevated levels of neutralizing antibodies.

These vaccines contain an antigen or an antigenic fraction with a major role in triggering the production of antibodies. Elimination of various cellular protein components, a nucleic acid, of no major importance for immunogenicity, causes a significant decrease in side effects. They have frequently used influenza vaccines with surface antigen types, hemagglutinin and neuraminidase (Influvac-Solvay Pharmaceuticals, FluarixGlaxoSmithKline) or those with fragments and virion (Vaxigrip-Sanofi Pasteur). The vaccines obtained are just as conventional by molecular recombination, such as those of hepatitis B second generation (Engerix B-GlaxoSmithKline; Euvax BSanofi Pasteur; Recombivax HB-Merck \& Co) or third generation, what contains recombinant DNA HBsAg [47].

\section{COVID-19 and normal vaccines development: will it make difference?}

\section{Normal vaccines development}

Vaccines are some of the most closely monitored and monitored biological drugs available [108]. The development of a vaccine is a complex process, which extends over very long 
periods and differs from the process of developing traditional medicines.

The rigors are higher than in the case of medicines because, unlike drugs, vaccines are, by definition, administered to a huge number of healthy people. Historically, there have been situations of contamination of vaccines with other viruses, fortunately without consequences [48]. Notably, one-third of polio vaccines administered in the USA between 1955 and 1963 also contained simian virus 40 (SV40), which was spread in complete ignorance through the manufacturing process. More recently, rotavirus vaccines have been discovered to have porcine circoviruses. To avoid such situations, anything to be tested on humans must first be checked for purity and then secured production lines.

\section{Vaccine development}

\section{Preclinical studies}

The first step in vaccine development is the preclinical development stage to determine the safety profile of the vaccine. The information collected from these studies is essential for the safe start of clinical trials in human subjects [49].

\section{Safety testing requirements}

- Phase I vaccine trials: Researchers are testing the candidate vaccine on a small number of volunteers $(20-50)$ to evaluate its safety, determine the dosage, and identify side effects. All these data are obtained by comparing the vaccine with control or inactive substance called placebo (e.g., saline). At this stage, data about the dose and the period between vaccinations necessary to ensure an optimal response of the immune system are identified.

- Phase II vaccine trials: Vaccines that have good results during phase I studies go into the second stage of studies. Here, the vaccine is given to a larger group of volunteers (100-300) to assess its safety and immunogenicity (the ability of a substance to produce an immune response). Also, at this stage, the correct dosage and administration schedule are explored in more detail. This phase can take two or more years.

- Phase III vaccine trials: The most promising candidate vaccines reach phase III of clinical trials, where 3000 50,000 volunteers are enrolled. This stage aims to measure the effectiveness of vaccines on a large scale among the population for which the vaccine is intended. At this stage, concomitant administration with other vaccines is also tested. Phase III of clinical trials may take 3-5 years.

- Approval by regulatory agencies, IND (Investigational New Drug) of the FDA (Food and Drug Administration) in the USA and the European Medicines Agency (EMA) in the European Union, after which the vaccine can be produced on a large scale and thus reach the population.

- Phase IV: Pharmacovigilance, monitoring the vaccine adverse event. Once arrived on the pharmaceutical market, the vaccine begins the process of pharmacovigilance. It involves strict monitoring of vaccines to detect, analyze, understand, prevent, and communicate any adverse effects following immunization or any other aspects related to the vaccine or immunization.

Long-term clinical trials are often performed to assess whether the protection provided by the vaccine is long-lasting. The process of developing and approving a vaccine takes more than a decade and is tested on tens of thousands of volunteers. Moreover, the safety and efficacy of vaccines are not only monitored by manufacturers but also by the competent authorities [50].

\section{COVID-19 vaccines development: recent progress}

The efforts of researchers around the world to obtain vaccines against SARS-CoV-2 as a therapeutic solution in the COVID19 pandemic have no precedent in terms of scale and speed.

As "speed" is the dominant feature of obtaining these vaccines, there is an indication that these vaccines may be available under emergency conditions or similar protocols by 2012. But this urgent need represents a fundamental change in the classic and traditional stages of vaccine development that last on average over 10 years, even compared with a period of 5 years necessary for Ebola vaccines[51].

This rapid and urgent need for the development of COVID19 vaccines involves new development concepts: (i) parallel and adaptive development phases, (ii) innovative regulatory processes, and (iii) large production capacity.

For the standard vaccines, in the preclinical stage, the technology that will be part of the vaccine is selected; the efficiency, safety on human cells (in vitro), and animal models (in vivo) are tested. If the in vitro anti-infective effect is expected, and many cells do not die excessively, in vivo animal studies are performed [52].

The preclinical stage lasts between $11 / 2$ [53] and $21 / 2$ [54] years and is by far the most selective; the estimate is that less than $20 \%$ [54] of the studies manage to pass the human test. Some studies fail because the product does not work, and others fail because they can no longer find funding. These aspects may be a reason for failure for potential anti-COVID vaccines announced by WHO as "preclinical testing" [39].

The second step is human testing and includes FDA (USA) and EMA (Europe) approvals [55, 56]. This stage is divided into 3 phases: testing on tens of people (phase I), testing on hundreds of people (phase II), and testing on thousands of people (phases III/IV) [57, 58]. 
Because the goal is to determine the dose at which the vaccine is effective, and especially to avoid side effects, it is always preferable to start small-scale testing [59].

Until now, only five vaccines skipped the preclinical stage and began to be administered directly to human subjects in phase I studies [60]. Two of them are developed by the American companies Moderna (RNA vaccine) [61] and Inovio (DNA vaccine) [62] and another three vaccine candidates by China, by CanSino Biologics (non-replicating adenoviral vector) [63], and Shenzhen GenoImmune Medical Institute (immune cells modified with a lentiviral vector) [64].

In light of these aspects, a possible diagram for COVID-19 vaccine development would be the following: (i) phases I and II lasting 6 months each; (ii) complete shunting, on emergency criteria of phase III; and (iii) 6 months for manufacturing and distribution, thus reaching a total of 18 months (Fig. 3).

An example of possible developing COVID-19 vaccine A manufacturer will contribute the antigen based on the S protein, which is based on recombinant DNA technology. This technology was able to genetically perfectly reproduce the proteins on the surface of the virus, and the DNA antigen sequence was mixed with the recombinant DNA on the baculovirus platform, this being the basic combination developed by Sanofi for their influenza vaccines. Another manufacturer will contribute the latest research in the field of adjuvant technology. The use of adjuvants can be especially important in a pandemic situation, as it can reduce the amount of protein needed for a dose, allowing more doses of vaccine to be produced and thus helping to protect more people [65]. The combination of protein-based antigen with an adjuvant is very well-known and used in many vaccines. Using an adjuvant in a vaccine has been shown to improve the immune response and have stronger and longer-lasting results than just using the vaccine alone. In addition, it can significantly improve the production of an effective, large-scale vaccine.

Given that the current pandemic situation is an extraordinary humanitarian and financial challenge, the companies consider global access to COVID-19 vaccines to be a priority at this time. The manufacturers are committed to making any vaccine developed through this collaboration accessible to the public, through a system that provides a fair distribution to people in all affected countries.

\section{Conclusions and future perspectives}

So far, there has been no pandemic coronavirus. The novel coronavirus 2019 is related to those that caused the epidemics of SARS (2002-2003) and MERS (2013). Unfortunately, there are no vaccines or vaccine candidates for SARS or MERS that could be converted for this pandemic. In addition, they could not have been used anyway, because it seems that the new virus is significantly different from previous coronaviruses.

Studies for the development of a COVID-19 vaccine are in various stages of development. Some methods use messenger RNA, others that use DNA, which is then translated, and specific immunogenic proteins are produced. Beyond the basic research that determines which antigens can cause a neutralizing immune response to the virus, two very important elements must be considered in the development of a vaccine. These are safety studies and efficacy studies. In the

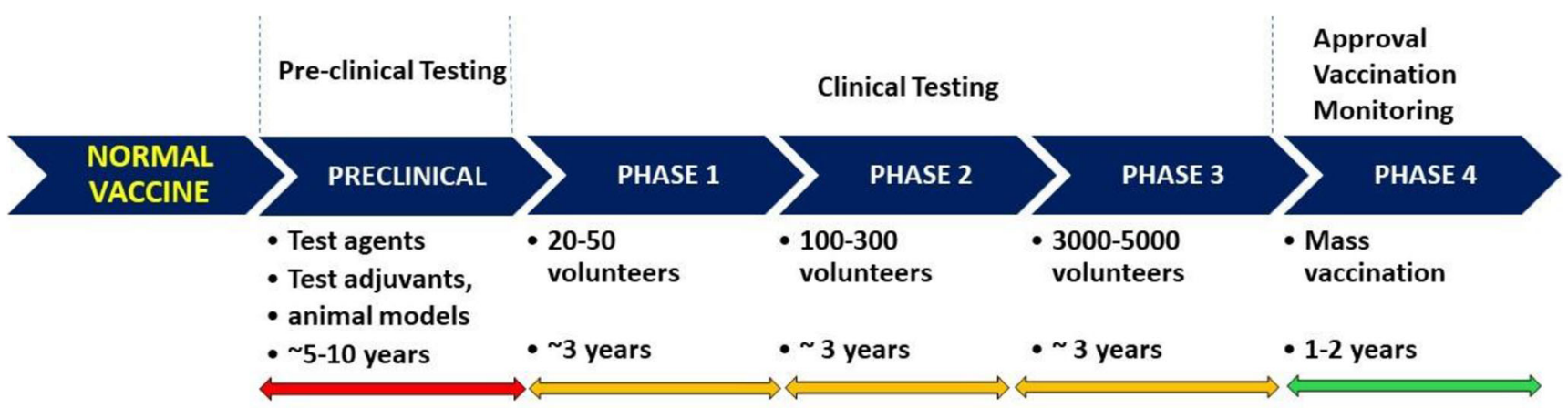

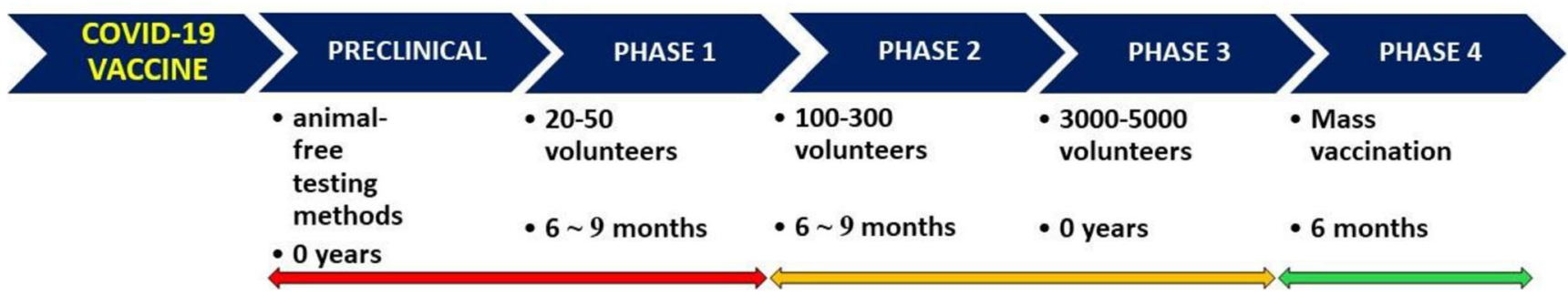

Fig. 3 A comparative scheme regarding the development of normal vaccines versus potential COVID-19 vaccines 
development of the vaccine, it is essential to consider the standard of the 3Rs: replacement, reduction, and refinement. In the case of standard vaccines, these fundamental properties are tested preclinically (in vitro and in vivo) and subsequently in human clinical trials, during many years of research. In vivo, vaccine testing is time-consuming, and most often, tests fail when the pathogens tested are not specific to humans.

If, during the previous SARS epidemic, it had ended, and we had now a vaccine approved for human use, all steps of the trial could probably be skipped safely, and this is supported by the biological similarity of coronaviruses large enough. Thus, if a SARS or MERS vaccine already existed, it could very easily have been adapted for SARS-CoV-2 without complex testing.

As a consequence, it is highly desirable to develop appropriate technologies that respond swiftly to control the current COVID-19 pandemic.

Availability of data and material Not applicable

\section{Compliance with ethical standards}

Conflicts of interest The authors declare that they have no conflict of interest.

Ethics approval Not applicable

Consent to participate Not applicable

Consent for publication Not applicable

Code availability Not applicable

\section{References}

1. Docea AO, Tsatsakis A, Albulescu D, Cristea O, Zlatian O, Vinceti $\mathrm{M}$, et al. A new threat from an old enemy: re-emergence of coronavirus. Int J Mol Med. 2020;45(6):1631-43.

2. Chen N, Zhou M, Dong X, Qu J, Gong F, Han Y, et al. Epidemiological and clinical characteristics of 99 cases of 2019 novel coronavirus pneumonia in Wuhan, China: a descriptive study. Lancet. 2020;395(10223):507-13.

3. Goumenou M, Sarigiannis D, Tsatsakis A, Anesti O, Docea AO, Petrakis D, et al. COVID-19 in Northern Italy: an integrative overview of factors possibly influencing the sharp increase of the outbreak. Mol Med Rep. 2020;22(1):20-32.

4. Tsatsakis A, Petrakis D, Nikolouzakis TK, Docea AO, Calina D, Vinceti M, et al. COVID-19, an opportunity to reevaluate the correlation between long-term effects of anthropogenic pollutants on viral epidemic/pandemic events and prevalence. Food Chem Toxicol. 2020;141:111418.

5. Adams ER, Augustin Y, Byrne RL, et al. Rapid development of COVID-19 rapid diagnostics for low resource settings: accelerating delivery through transparency, responsiveness, and open collaboration. medRxiv; 2020. https://doi.org/10.1101/2020.04.29. 20082099 .
6. Hsueh P-R, Huang LM, Chen PJ, Kao CL, Yang PC. Chronological evolution of $\operatorname{IgM}$, IgA, IgG and neutralisation antibodies after infection with SARS-associated coronavirus. Clin Microbiol Infect. 2004;10(12):1062-6.

7. Beavis KG, Matushek SM, Abeleda APF, Bethel C, Hunt C, Gillen $\mathrm{S}$, et al. Evaluation of the EUROIMMUN anti-SARS-CoV-2 ELISA assay for detection of IgA and IgG antibodies. J Clin Virol. 2020;129:104468.

8. Ayouba A, Thaurignac G, Morquin D, Tuaillon E, Raulino R, Nkuba A, et al. Multiplex detection and dynamics of IgG antibodies to SARS-CoV2 and the highly pathogenic human coronaviruses SARS-CoV and MERS-CoV. J Clin Virol. 2020;129:104521.

9. Ju B, Zhang Q, Ge J, Wang R, Sun J, Ge X, et al. Human neutralizing antibodies elicited by SARS-CoV-2 infection. Nature. 2020;584(7819):115-9. https://doi.org/10.1038/s41586-020-2380$\mathrm{z}$.

10. Wang $\mathrm{C}$, et al. A human monoclonal antibody blocking SARSCoV-2 infection. Nat Commun. 2020;11(1):1-6.

11. OKBA NM, Muller MA, Li W, et al. SARS-CoV-2 specific antibody responses in COVID-19 patients. medRxiv; 2020. https://doi. org/10.1101/2020.03.18.20038059.

12. Tsang JS, Dobaño C, VanDamme P, Moncunill G, Marchant A, Othman RB, et al. Improving vaccine-induced immunity: can Baseline Predict Outcome? Trends Immunol. 2020;41:457-65.

13. Jiang S, Hillyer C, Du L. Neutralizing antibodies against SARSCoV-2 and other human Coronaviruses. Trends Immunol. 2020;41(5):355-9. https://doi.org/10.1016/j.it.2020.03.007.

14. Lurie N, Saville M, Hatchett R, Halton J. Developing Covid-19 vaccines at pandemic speed. N Engl J Med. 2020;382(21):1969-73.

15. Yaqinuddin A. Cross-immunity between respiratory coronaviruses may limit COVID-19 fatalities. Med Hypotheses. 2020;144: 110049.

16. Caturegli G, Materi J, Howard BM, Caturegli P. Clinical validity of serum antibodies to SARS-CoV-2: a case-control study. Ann Intern Med. 2020;6:M20-2889. https://doi.org/10.7326/M20-2889.

17. Tetro JA. Is COVID-19 receiving ADE from other coronaviruses? Microbes Infect. 2020;22(2):72-3.

18. Calina D, Docea A, Petrakis D, Egorov A, Ishmukhametov A, Gabibov A, et al. Towards effective COVID-19 vaccines: updates, perspectives and challenges. Int J Mol Med. 2020;46(1):3-16.

19. Tomljenovic L, Shaw CA. "One-size fits all"? Vaccine. 2012;30(12):2040. https://doi.org/10.1016/j.vaccine.2011.11.053.

20. Kissler SM, Tedijanto C, Goldstein E, Grad YH, Lipsitch M. Projecting the transmission dynamics of SARS-CoV-2 through the postpandemic period. Science. 2020;368(6493):860-8.

21. Grifoni A, Weiskopf D, Ramirez SI, et al. Targets of T cell responses to SARS-CoV-2 Coronavirus in humans with COVID-19 disease and unexposed individuals. Cell. 2020;181(7):1489501.e15. https://doi.org/10.1016/j.cell.2020.05.015.

22. Calina D, Docea A, Petrakis D, Egorov A, Ishmukhametov A, Gabibov A, et al. Towards effective COVID-19 vaccines: updates, perspectives and challenges (Review). Int J Mol Med. 2020;46(1): $3-16$.

23. McAnulty JM, et al. Vaccine failures and vaccine effectiveness in children during measles outbreaks in New South Wales, MarchMay 2006. Commun Dis Intell Q Rep. 2009;33(1):21.

24. Haralambieva IH, Ovsyannikova IG, Pankratz VS, Kennedy RB, Jacobson RM, Poland GA. The genetic basis for interindividual immune response variation to measles vaccine: new understanding and new vaccine approaches. Expert Rev Vaccines. 2013;12(1):5770 .

25. Kudrat-E-Zahan M, et al. A perspective on emerging therapeutic interventions for COVID-19.

26. Wiedermann U, Garner-Spitzer E, Wagner A. Primary vaccine failure to routine vaccines: why and what to do? Hum Vaccin Immunother. 2016;12(1):239-43. 
27. Osterholm MT, Kelley NS, Sommer A, Belongia EA. Efficacy and effectiveness of influenza vaccines: a systematic review and metaanalysis. Lancet Infect Dis. 2012;12(1):36-44.

28. Wu F, et al. Neutralizing antibody responses to SARS-CoV-2 in a COVID-19 recovered patient cohort and their implications. medRxiv. 2020; 2020-033020047365. https://doi.org/10.1101/ 2020.03.30.20047365. Accessed 2020-04-29.

29. Peeples L. News feature: avoiding pitfalls in the pursuit of a COVID-19 vaccine. Proc Natl Acad Sci. 2020;117(15):8218-21.

30. Davidkin I, Jokinen S, Broman M, Leinikki P, Peltola H. Persistence of measles, mumps, and rubella antibodies in an MMR-vaccinated cohort: a 20-year follow-up. J Infect Dis. 2008;197(7):950-6.

31. National Academies of Sciences, E. and Medicine, Integrating clinical research into epidemic response: the Ebola experience. National Academies Press; 2017.

32. Zhu F-C, Wurie AH, Hou LH, Liang Q, Li YH, Russell JBW, et al. Safety and immunogenicity of a recombinant adenovirus type- 5 vector-based Ebola vaccine in healthy adults in Sierra Leone: a single-centre, randomised, double-blind, placebo-controlled, phase 2 trial. Lancet. 2017;389(10069):621-8.

33. Callow KA, Parry HF, Sergeant M, Tyrrell DAJ. The time course of the immune response to experimental coronavirus infection of man. Epidemiol Infect. 1990;105(2):435-46.

34. Cao W-C, Liu W, Zhang PH, Zhang F, Richardus JH. Disappearance of antibodies to SARS-associated coronavirus after recovery. N Engl J Med. 2007;357(11):1162-3.

35. Wu LP, Wang NC, Chang YH, Tian XY, Na DY, Zhang LY, et al. Duration of antibody responses after severe acute respiratory syndrome. Emerg Infect Dis. 2007;13(10):1562-4.

36. Payne DC, Iblan I, Rha B, Alqasrawi S, Haddadin A, al Nsour M, et al. Persistence of antibodies against Middle East respiratory syndrome coronavirus. Emerg Infect Dis. 2016;22(10):1824-6.

37. Poutanen SM. Human Coronaviruses. Principles and Practice of Pediatric Infectious Diseases. 2018;1148-52.e3. https://doi.org/10. 1016/B978-0-323-40181-4.00222-X.

38. Alshukairi AN, et al. Antibody response and disease severity in healthcare worker MERS survivors. Emerg Infect Dis. 2016;22(6):1113-5.

39. Organization, W.H., DRAFT landscape of COVID-19 candidate vaccines. World. 2020.

40. Marston HD, Paules CI, Fauci AS. The critical role of biomedical research in pandemic preparedness. JAMA. 2017;318(18):1757-8.

41. Pardi N, Hogan MJ, Porter FW, Weissman D. mRNA vaccines - a new era in vaccinology. Nat Rev Drug Discov. 2018;17(4):261-79.

42. Rauch S, Jasny E, Schmidt KE, Petsch B. New vaccine technologies to combat outbreak situations. Front Immunol. 2018;9:1963.

43. Bliss CM, Bowyer G, Anagnostou NA, Havelock T, Snudden CM, Davies $\mathrm{H}$, et al. Assessment of novel vaccination regimens using viral vectored liver stage malaria vaccines encoding ME-TRAP. Sci Rep. 2018;8(1):3390.

44. Ewer K, Rampling T, Venkatraman N, Bowyer G, Wright D, Lambe T, et al. A monovalent chimpanzee adenovirus Ebola vaccine boosted with MVA. N Engl J Med. 2016;374(17):1635-46.

45. Callaway $\mathrm{E}$. The race for coronavirus vaccines: a graphical guide. Nature. 2020;580(7805):576-7.
46. Yang L, Tian D, Liu W. Strategies for vaccine development of COVID-19. Sheng Wu Gong Cheng Xue Bao. 2020;36(4):593.

47. Khuroo MS, Khuroo M, Khuroo MS, Sofi AA, Khuroo NS. COVID-19 Vaccines: A Race Against Time in the Middle of Death and Devastation! J Clin Exp Hepatol. 2020. https://doi.org/ 10.1016/j.jceh.2020.06.003.

48. Petricciani J, Sheets R, Griffiths E, Knezevic I. Adventitious agents in viral vaccines: lessons learned from 4 case studies. Biologicals. 2014;42(5):223-36.

49. Agency, E.M., Guideline on quality, non-clinical and clinical requirements for investigational advanced therapy medicinal products in clinical trials. 2020.

50. De Mattia F, et al. The consistency approach for quality control of vaccines-a strategy to improve quality control and implement 3Rs. Biologicals. 2011;39(1):59-65.

51. Powlson J, et al. Characterization of antigenic MHC-class-Irestricted t cell epitopes in the glycoprotein of ebolavirus. Cell Rep. 2019;29(9):2537-2545.e3.

52. Daneshian M, Busquet F, Hartung T, Leist M. Animal use for science in Europe. Alternatives to animal experimentation. ALTEX. 2015;32(4):261-74.

53. Pronker ES, Weenen TC, Commandeur H, Claassen EHJHM, Osterhaus ADME. Risk in vaccine research and development quantified. PLoS One. 2013;8(3):e57755

54. Struck M-M. Vaccine R\&D success rates and development times. Nat Biotechnol. 1996;14(5):591-3.

55. FDA, Investigational new drug (IND) Application. 2020.

56. Agency, E.M., Clinical Trial Regulation. 2019.

57. Bulik NB, et al. Reactogenicity and medically attended adverse events following hexavalent vaccination: an observational prospective study. Farmacia. 2019;67(6):1018-24.

58. Bucsa $\mathrm{C}$, et al. Adverse events following measles-mumps-rubella vaccination. A prospective observational study. Farmacia. 2020;68(2):219-24.

59. Calina D, Hartung T, Docea AO, et al. COVID-19 vaccines: ethical framework concerning human challenge studies. DARU J Pharm Sci 2020;1-6. https://doi.org/10.1007/s40199-020-00371-8.

60. Le TT, et al. The COVID-19 vaccine development landscape. Nat Rev Drug Discov. 2020;19(5):305-6.

61. Medicine, N.L.o., Safety and immunogenicity study of 2019-nCoV vaccine (mRNA-1273) for prophylaxis SARS CoV-2 infection (COVID-19). 2020.

62. Medicine, N.L.o., Safety, tolerability and immunogenicity of INO4800 for COVID-19 in healthy volunteers. 2020.

63. Medicine, N.L.o., Phase I clinical trial of a COVID-19 vaccine in 18-60 healthy adults (CTCOVID-19). 2020.

64. Medicine, N.L.o., Immunity and safety of Covid-19 synthetic minigene vaccine. 2020.

65. Arsene AL, et al. A new era for the therapeutic management of the ongoing COVID-19 pandemic. Farmacia. 2020;68(2):185-96.

Publisher's note Springer Nature remains neutral with regard to jurisdictional claims in published maps and institutional affiliations. 